\title{
Delayed osteomyelitis resulting from an extension injury of the cervical spine: case report
}

\author{
Victoria Palmer, MPH, ${ }^{1}$ Randolph B. Cohen, MD, ${ }^{2}$ Bruce Braffman, MD, ${ }^{3}$ \\ Douglas L. Brockmeyer, MD, ${ }^{4}$ and Heather S. Spader, MD
}

\begin{abstract}
${ }^{1}$ Keiser University Physician Assistant Program, Ft. Lauderdale, Florida; ${ }^{2}$ Department of Pediatric Orthopedic Surgery, Joe DiMaggio Children's Hospital, Hollywood, Florida; ${ }^{3}$ Department of Diagnostic Imaging, Memorial Regional Hospital, Hollywood, Florida; ${ }^{4}$ Department of Pediatric Neurosurgery, University of Utah, Salt Lake City, Utah; and ${ }^{5}$ Division of Pediatric Neurosurgery, Joe DiMaggio Children's Hospital, Hollywood, Florida
\end{abstract}

Osteomyelitis of the cervical spine is an exceedingly rare lesion in pediatric practice and is caused by a variety of mechanisms. The authors present a case in which cervical osteomyelitis presented in a delayed manner after the patient experienced a stable cervical extension injury at the C4-5 level. On review of the original images, the authors noted a likely perforation in the retropharyngeal space. This case highlights the risk of retropharyngeal injury in cervical trauma, with the seldom-seen complication of osteomyelitis as a result.

https://thejns.org/doi/abs/10.3171/2017.5.PEDS1777

KEY WORDS osteomyelitis; cervical spine trauma; pediatric; infection

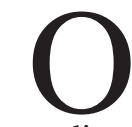
STEOMYELITIS of the cervical spine is a rare condition, representing only $3 \%-6 \%$ of all cases of vertebral osteomyelitis. ${ }^{10}$ Vertebral osteomyelitis in pediatric patients is rarer still, representing only $1 \%-2 \%$ of all children with osteomyelitis. ${ }^{4}$ The most common cause of cervical osteomyelitis is hematogenous seeding from a distant source of infection; ${ }^{12}$ however, direct extension of the infection from adjacent prevertebral spaces leads to osteomyelitis in $33 \%$ of the cases. ${ }^{2,5}$

Previous case reports have demonstrated that cervical spondylodiscitis and cervical spinal epidural abscess have arisen from minor trauma and injury to the posterior pharyngeal mucosa after ingestion of a foreign body.,14 $\mathrm{In}$ these cases, microperforation of the posterior pharyngeal wall served as a source of infection to the retropharyngeal space and, consequently, the cervical spine.

Additionally, high-energy trauma to the cervical spine caused by hyperextension injuries has been shown to result in retropharyngeal and paravertebral soft-tissue injury in the pediatric population. ${ }^{1,8}$ Classically, rear end-impact car crashes or contact sport injuries are the causative mechanism. The injury can cause esophageal perforation and microrupture of the retropharyngeal mucosa, thereby acting as a source of infection to the prevertebral space. ${ }^{13}$

To date, to the best of our knowledge, there have been no previously reported cases of cervical osteomyelitis af- ter cervical trauma and pharyngeal soft-tissue injury in the pediatric population. We describe an unusual case in which delayed cervical osteomyelitis and epidural abscess were seen after a pharyngeal soft-tissue injury due to cervical trauma in an adolescent patient. Because of the risk of delayed diagnosis, clinicians should be aware of the potential for infections of the cervical spine after a cervical injury associated with retropharyngeal tissue disruption and dysphagia.

\section{Case Report}

Presentation and Initial Management

A previously healthy 15 -year-old boy sustained an injury to his neck during a basketball game when another player fell on top of him, causing sudden forceful extension of the cervical spine. He reported significant neck pain at the time of the injury and two episodes of vomiting the next day. He also complained of dysphagia approximately 8-16 hours after the injury. Twenty-four hours after injury, he had an uncomfortable sensation when swallowing, so he could only drink liquids. When he ate solid food, it pressed against his neck and made him uncomfortable, but he did not have significant pain. After findings on radiographs obtained at an outside emergency department were interpreted as normal, he was discharged to home 
and prescribed $600 \mathrm{mg}$ of ibuprofen and $5 \mathrm{mg}$ of cyclobenzaprine. No cervical collar was placed at this time.

Three days after the injury, the patient was seen at our emergency department complaining of persistent, worsening neck pain and dysphagia. Neck movement was painful and restricted in all directions. There was no tenderness over the cervical spine or surrounding musculature and no focal neurological findings. Magnetic resonance imaging (MRI) (sagittal T1-weighted, T2-weighted, and short-tau inversion-recovery [STIR] images without contrast) of the cervical spine revealed prevertebral soft-tissue swelling extending from C-2 to C-5. Mild separation of the C4-5 disc from the vertebral body and disruption of a small area of the anterior longitudinal ligament along the superior aspect of C-5 were suggestive of an injury along the $\mathrm{C} 4-5$ disc space. There was no evidence of fracture or malalignment of the vertebral bodies, and no spinal cord compression or intraspinal abnormalities were identified. Retropharyngeal injury was not reported at the time of this MRI session. The patient's neck was immobilized with a cervical collar, and he was discharged and prescribed analgesic medications (acetaminophen-codeine 300-mg/30mg tablets).

After 7-8 days, the dysphagia resolved and did not return. During evaluation by pediatric neurosurgery 17 days after discharge, the patient's neck pain had also resolved. He exhibited posterior neck stiffness and decreased extension of the cervical spine. Neck movement was otherwise intact, and there was no edema, crepitus, or tenderness along the cervical spine. The findings of a neurological examination were normal. Flexion-extension radiographs showed no instability (Fig. 1). Physical examination during reassessment 31 days after discharge revealed improved range of motion with extension, but the patient had remaining tightness with cervical spine rotation. He did not demonstrate any additional deficits during the followup examination, so the cervical collar was removed and the patient was released from neurosurgical care.

\section{Presentation With Abscess}

Nine months after the initial injury, the patient presented to a pediatric orthopedic surgery office complaining of increasing pain in the cervical spine accompanied by left shoulder pain. This pain had persisted for several weeks and was moderately relieved with ketorolac and diazepam. He denied any numbness, tingling, or changes in bowel or bladder control. Physical examination revealed tenderness of the cervical paravertebral musculature and increased pain with hyperextension and forward flexion of the cervical spine. Neck flexion, extension, lateral side bending, and rotation were restricted.

A standing, anteroposterior radiograph of the cervical spine revealed disc space narrowing and endplate irregularity at the C4-5 level. No retrolisthesis or anterolisthesis was noted. MRI of the cervical spine revealed C4-5 discitis/osteomyelitis with cortical endplate destruction and a paraspinal/epidural phlegmon, but no spinal cord compression was noted (Fig. 2). The previous MR images obtained at the time of the injury were reviewed again. Sagittal STIR MRI without contrast showed a disruption in the cervical C4-5 disc space, a small tear in the anterior
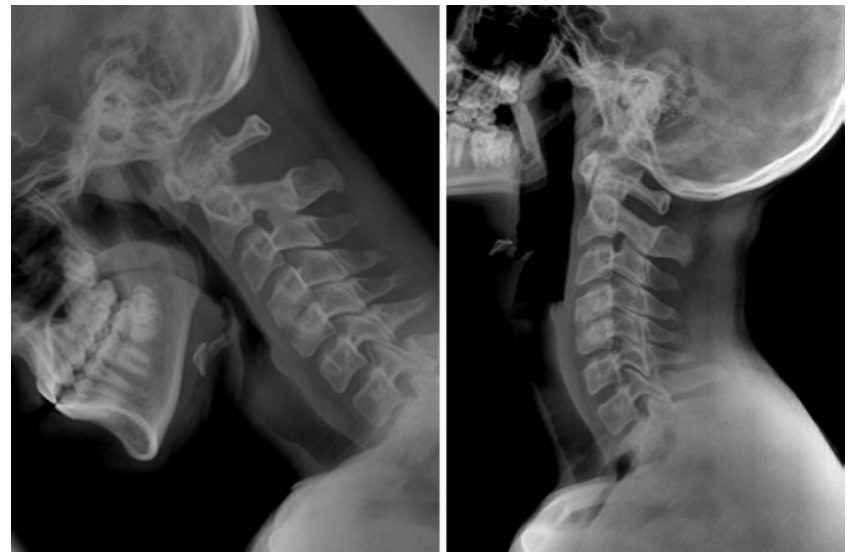

FIG. 1. Flexion and extension cervical spine radiographs showing no significant movement.

longitudinal ligament, and edema and/or a small perforation in the retropharyngeal space (Fig. 3).

Based on the MRI results, the patient was advised to go to the emergency department for further evaluation. Laboratory workup showed a normal white blood cell count (7190/ $\mu \mathrm{l}$; reference range $4500-11,000 / \mu \mathrm{l})$, an elevated erythrocyte sedimentation rate (ESR) $(19 \mathrm{~mm} /$ hour; reference range $0-10 \mathrm{~mm} /$ hour), and an elevated C-reactive protein (CRP) $(1.0 \mathrm{mg} / \mathrm{dl}$; reference range $0.00-0.3 \mathrm{mg} / \mathrm{dl})$. Blood cultures taken at this time were negative. An esophagram was performed, and no areas of leakage were identified. Because the most likely mechanism of bacterial spread was deemed to be a microperforation of the pharynx at the time of the injury that seeded the bone, endoscopy was not thought to be necessary since a mucosal perforation this small would have healed completely after 9 months. The patient was evaluated for a biopsy of the lesion; however, given the proximity to the carotid artery, esophagus, and trachea, the interventional radiologist and otolaryngologist did not do a needle biopsy because they believed that the risk of procedure was not acceptable in the setting of an osteomyelitis likely caused by oral flora that would therefore likely be responsive to standard antibiotic treatment. We thought that the collection was not large enough to warrant an open biopsy. Therefore, the patient was empirically placed on ampicillin-sulbactam (3 g intravenously every 6 hours) and vancomycin (1300 mg intravenously every 8 hours) and admitted to the pediatric intensive care unit for observation. A soft collar was placed for comfort. He exhibited improvement in the intensive care unit and was transferred to the floor after 1 day, where he made continued improvement in his symptoms. An echocardiogram was obtained to rule out cardiac vegetations. The patient was discharged on the 4th day of hospitalization. A 6-week course of intravenous antibiotics was recommended in the form of linezolid and ceftriaxone.

\section{Follow-Up}

On follow-up 1 month postdischarge, the patient had full range of motion of the cervical spine with minimal pain. His extension was improved, but he was still missing $15^{\circ}$ of full terminal extension. Upper-extremity 

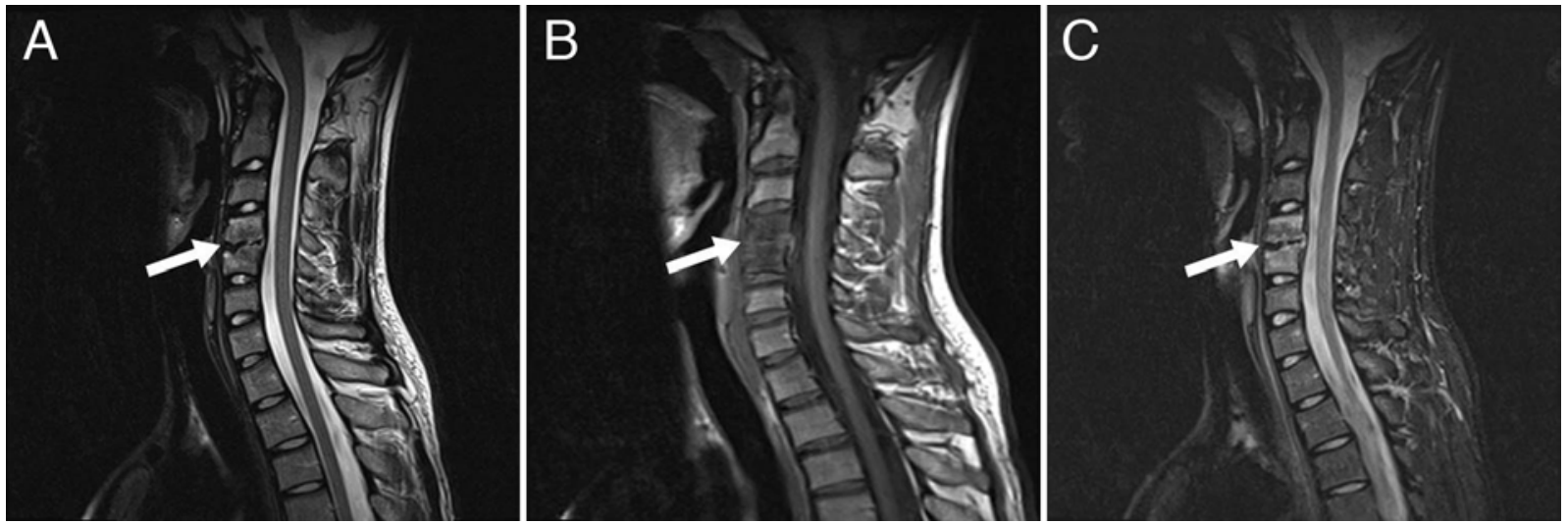

FIG. 2. Sagittal T1-weighted (A), T2-weighted (B), and STIR (C) images without contrast. A C4-5 discitis/osteomyelitis with cortical endplate destruction is seen (arrow).

strength was normal, as were biceps and triceps reflexes. Two weeks later, cervical spine plain radiographs showed obliteration of the disc space between C- 4 and C-5 but no change in disc heights in adjacent vertebrae. There was no antero- or retrolisthesis and improved alignment was observed. MRI of the cervical spine with and without contrast showed further loss of vertebral body height but resolved paraspinal/epidural phlegmon (Fig. 4). Physical examination revealed full range of motion of the cervical spine, including extension, so the use of the collar was discontinued. Four months later, a flexion-extension radiography show autofusion of C-4 and C-5 (Fig. 5).

\section{Discussion}

This case demonstrates a rare example of a pediatric cervical injury that likely led to delayed cervical osteo- myelitis from a small pharyngeal perforation that seeded the bone and eventually developed into osteomyelitis. Extension injuries of the cervical spine commonly involve the anterior longitudinal ligament, disc space, and, occasionally, the posterior longitudinal ligament $;{ }^{6}$ however, infectious seeding of the cervical spine from traumatic retropharyngeal injuries, while previously described, is far less common. ${ }^{5,7,14}$ The presentation and clinical course of cervical osteomyelitis after cervical trauma is described to increase awareness of this rare complication.

\section{Epidemiology of Pediatric Vertebral Osteomyelitis}

The main mechanism of osteomyelitic spread is hematogenous $(50 \%)$, followed by direct extension (33\%) and inoculation from spinal procedures (15\%). ${ }^{5}$ The most common bacterium identified is Staphylococcus aureus
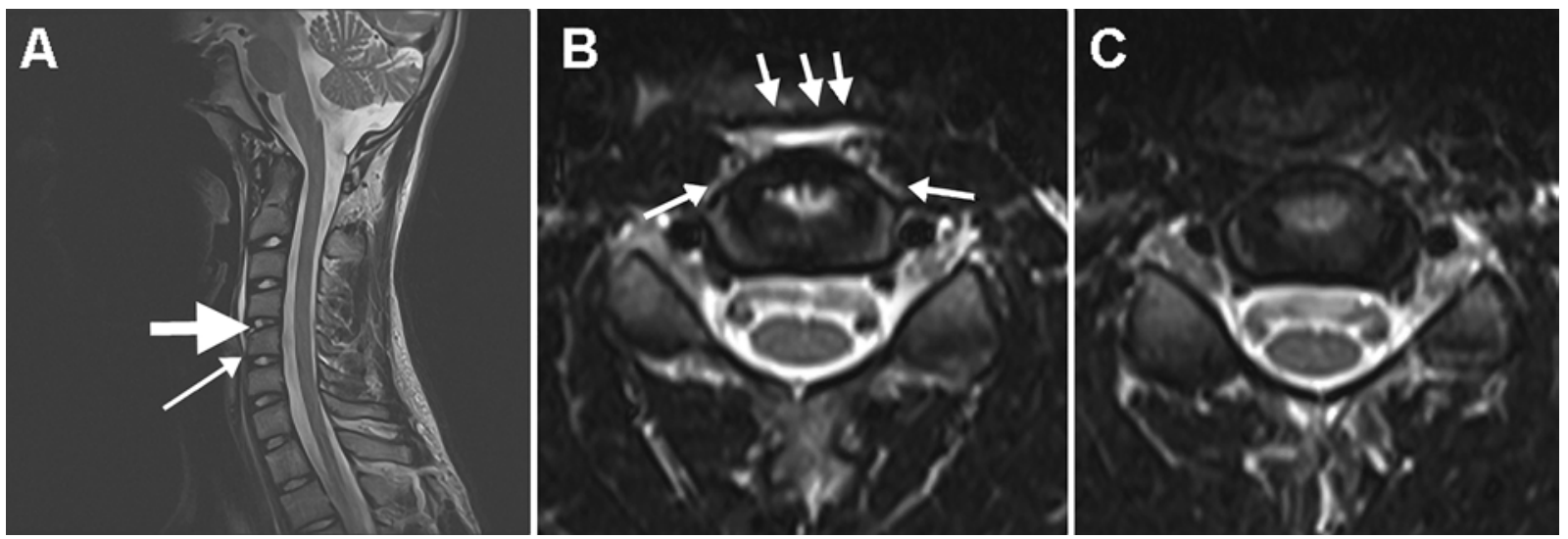

FIG. 3. A: Sagittal T2-weighted MR image of the cervical spine without contrast enhancement. The obliquely oriented hyperintense signal extends from the inferior portion of the $\mathrm{C} 4-5$ intervertebral disc into the thinned and partially torn anterior longitudinal ligament (thick arrow). The hyperintense signal anterior to the C3-5 vertebrae (fluid and/or edema) is in either the prevertebral space or the retropharyngeal space (thin arrow). This is indeterminate on sagittal image, but more visible on axial images. B: Axial T2-weighted MR image of the cervical spine without contrast at the C4-5 level. Hyperintense signal (fluid and/or edema) is in both the retropharyngeal space anterior to the paired longus colli muscles (three arrows) and in the prevertebral space, between the anterior margin of the intervertebral disc and the paired longus colli muscles (single arrow, one on each side), suggestive of a perforation. C: Axial T2-weighted MR image of the cervical spine without contrast at the C5-6 level. In contrast to the level above, at this level there is no abnormal fluid or edema in either the retropharyngeal space or the prevertebral space. There is normal signal anterior and posterior to the longus colli muscles. 


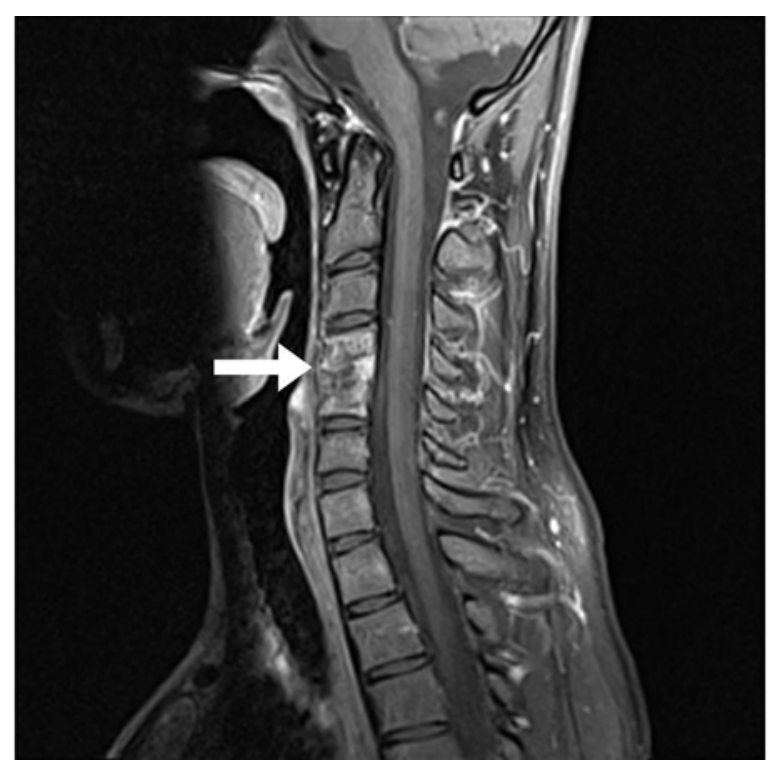

FIG. 4. Sagittal T1 contrast MR image of the cervical spine after treatment (arrow indicates autofusion of C-4 and C-5).

$(63 \%-80 \%) .{ }^{9}$ In children younger than 5 years of age, infections tend to first present in the disc space because of the vascular channels in the cartilaginous regions of the disc space that involute by adolescence. The mechanism of infection in older children is thought to be hematogenous spread via end arteries that supply the subchondral spongy

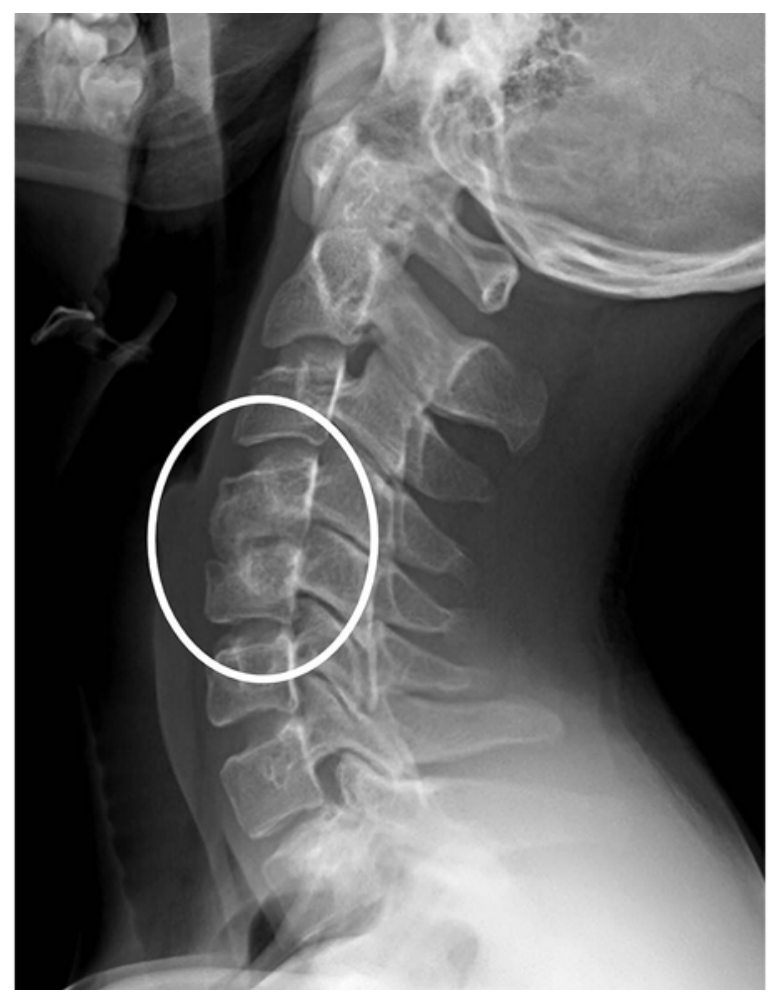

FIG. 5. Flexion-extension lateral cervical spine radiograph 5 months after treatment showing autofusion (circle). bone. The infection then seeds the bone and spreads by direct extension. ${ }^{9}$

\section{Diagnosis}

Laboratory tests for osteomyelitis are often not helpful. Fernandez et al. ${ }^{4}$ found that in patients with pediatric vertebral osteomyelitis, the mean white blood cell count was only $12,600 \mu$, the mean ESR was slightly elevated at $45.6 \mathrm{~mm} /$ hour, and the mean CRP was normal to slightly elevated. Unfortunately, blood cultures and local biopsies are often negative. ${ }^{3}$ In the adult population, the procalcitonin level has been found to be more reliable than ESR and CRP, but this finding has not yet been replicated in pediatric patients. ${ }^{4}$ Therefore, the best diagnostic modality is often imaging, specifically MRI scans. MRI has a sensitivity of $96 \%$, specificity of $93 \%$, and accuracy of 94\% compared with $82 \%$ sensitivity, $57 \%$ specificity, and $73 \%$ accuracy for radiography in diagnosing an epidural abscess or osteomyelitis. ${ }^{4}$

\section{Treatment}

Because vertebral osteomyelitis in children is not common, there are no standard guidelines for treatment. Therefore, the current gold standard is the same as with any pediatric osteomyelitis, namely, empirical treatment with antibiotics, first broadly and then narrowed, if a causative agent is isolated. If a bacterium is not isolated, then broad antibiotics are continued. ${ }^{3}$ If the treatment does not respond to antibiotics, then a surgical debridement can be done, but this is not the preferred initial treatment. ${ }^{3}$

\section{Retropharyngeal Perforations Causing Cervical Osteomyelitis}

Although unusual, cervical vertebral osteomyelitis is a known complication of retropharyngeal perforations. Jeon et al. ${ }^{7}$ reported a cervical abscess that was caused by ingestion of a fish bone. In a study by Goulart et al., ${ }^{5}$ the authors reported a large retropharyngeal abscess that evolved into a spinal osteomyelitis and epidural abscess after high-energy trauma and an unrecognized unstable cervical injury. Wadie et al. ${ }^{14}$ reported the case of a patient with a microperforation of the posterior pharyngeal wall caused by a pin, with no mucosal tear, who presented over a month later with osteomyelitis.

Retropharyngeal abscesses most commonly form from bacteria originating in the nasopharynx, middle ear, sinuses, or tonsils. The lymph nodes in this area degenerate during adolescence so there is a high incidence of spontaneous retropharyngeal abscesses in the adult population. The anatomy of the retropharyngeal space is defined by anterior and posterior compartments. These compartments are separated by alar fascia that derives from the deep cervical fascia. This fascia extends anteriorly from the skull base to the T-4 vertebra. Posteriorly, it extends down to the diaphragm. ${ }^{5}$ Between the alar fascia and prevertebral fascia is a space referred to as the "danger space" because it is an important potential pathway for spread of cranial or cervical pathology such as hemorrhage or infection. ${ }^{1}$

\section{Cervical Injuries Causing Retropharyngeal Perforation}

Cervical injuries causing subsequent injury to the ret- 
ropharyngeal space have been reported in the literature as well. In 1980, Stringer et al. ${ }^{11}$ described a cervical hyperextension injury that caused an esophageal perforation. Nurata et al. ${ }^{8}$ described a retropharyngeal hematoma and widened prevertebral space secondary to a whiplash injury. Anagnostara et al. ${ }^{1}$ described a retropharyngeal hematoma and prevertebral edema caused by whiplash. Of note, in a retrospective review, approximately $10 \%$ of patients with a spinal epidural abscess had a history of extraspinal or spinal trauma. ${ }^{5}$ In addition, the cervical injuries associated with retropharyngeal perforation tend to involve the anterior longitudinal ligament or anterior annulus. ${ }^{5}$

\section{Cervical Injury, Retropharyngeal Injury, and Vertebral Osteomyelitis}

This case combines an anterior cervical extension injury in which there was a known tear of the anterior longitudinal ligament, retropharyngeal edema, and likely a small pharyngeal perforation with a delayed osteomyelitis. Given the evidence that there is an association between retropharyngeal microperforations and osteomyelitis, when a cervical injury involves enough force to cause a disruption of the anterior longitudinal ligament, anterior annulus fibrosis, and retropharyngeal space, and when the patient has dysphagia after the injury, suspicion should be high for a pharyngeal injury. Further evaluation should include an esophagram and possibly endoscopy to rule out a perforation so that a decision can be made at the time of injury about whether antibiotics are needed for effective treatment and prevention of osteomyelitis.

\section{Acknowledgments}

We would like to thank Kristin Kraus, MSc, and Stephanie Eyerly-Webb, $\mathrm{PhD}$, for their editorial assistance with this paper.

\section{References}

1. Anagnostara A, Athanassopoulou A, Kailidou E, Markatos A, Eystathidis A, Papageorgiou S: Traumatic retropharyngeal hematoma and prevertebral edema induced by whiplash injury. Emerg Radiol 11:145-149, 2005

2. Beronius M, Bergman B, Andersson R: Vertebral osteomyelitis in Göteborg, Sweden: a retrospective study of patients during 1990-95. Scand J Infect Dis 33:527-532, 2001

3. Castellazzi L, Mantero M, Esposito S: Update on the management of pediatric acute osteomyelitis and septic arthritis. Int J Mol Sci 17:17, 2016
4. Fernandez M, Carrol CL, Baker CJ: Discitis and vertebral osteomyelitis in children: an 18-year review. Pediatrics 105:1299-1304, 2000

5. Goulart CR, Mattei TA, Fiore ME, Thoman WJ, Mendel E: Retropharyngeal abscess with secondary osteomyelitis and epidural abscess: proposed pathophysiological mechanism of an underrecognized complication of unstable craniocervical injuries: case report. J Neurosurg Spine 24:197-205, 2016

6. Greenberg M: Handbook of Neurosurgery, ed 7th. New York: Thieme, 2010

7. Jeon SH, Han DC, Lee SG, Park HM, Shin DJ, Lee YB: Eikenella corrodens cervical spinal epidural abscess induced by a fish bone. J Korean Med Sci 22:380-382, 2007

8. Nurata H, Yilmaz MB, Borcek AO, Oner AY, Baykaner MK: Retropharyngeal hematoma secondary to whiplash injury in childhood: a case report. Turk Neurosurg 22:521-523, 2012

9. Principi N, Esposito S: Infectious discitis and spondylodiscitis in children. Int J Mol Sci 17:539, 2016

10. Schimmer RC, Jeanneret C, Nunley PD, Jeanneret B: Osteomyelitis of the cervical spine: a potentially dramatic disease. J Spinal Disord Tech 15:110-117, 2002

11. Stringer WL, Kelly DL Jr, Johnston FR, Holliday RH: Hyperextension injury of the cervical spine with esophageal perforation. Case report. J Neurosurg 53:541-543, 1980

12. Tay BK, Deckey J, Hu SS: Spinal infections. J Am Acad Orthop Surg 10:188-197, 2002

13. Uhrenholt L, Freeman MD, Jurik AG, Jensen LL, Gregersen $\mathrm{M}$, Boel LW, et al: Esophageal injury in fatal rear-impact collisions. Forensic Sci Int 206:e52-e57, 2011

14. Wadie GM, Konefal SH, Dias MA, McLaughlin MR: Cervical spondylodiscitis from an ingested pin: a case report. J Pediatr Surg 40:593-596, 2005

\section{Disclosures}

The authors report no conflict of interest concerning the materials or methods used in this study or the findings specified in this paper.

\section{Author Contributions}

Conception and design: Spader, Cohen. Analysis and interpretation of data: all authors. Drafting the article: Spader, Palmer. Critically revising the article: all authors. Reviewed submitted version of manuscript: Spader. Approved the final version of the manuscript on behalf of all authors: Spader.

\section{Correspondence}

Heather Spader, Division of Pediatric Neurosurgery, Joe DiMaggio Children's Hospital, 1150 N 35th Ave., Ste. 205A, Hollywood, FL 33021. email: hspader@mhs.net. 